\title{
CRITICAL BLOW-UP AND EXTINCTION EXPONENTS FOR NON-NEWTON POLYTROPIC FILTRATION EQUATION WITH SOURCE
}

\author{
Jun Zhou And Chunlai Mu
}

\begin{abstract}
This paper deals with the critical blow-up and extinction exponents for the non-Newton polytropic filtration equation. We reveals a fact that the equation admits two critical exponents $q_{1}, q_{2} \in(0,+\infty)$ with $q_{1}<q_{2}$. In other words, when $q$ belongs to different intervals $\left(0, q_{1}\right),\left(q_{1}, q_{2}\right),\left(q_{2},+\infty\right)$, the solution possesses complete different properties. More precisely speaking, as far as the blow-up exponent is concerned, the global existence case consists of the interval $\left(0, q_{2}\right]$. However, when $q \in\left(q_{2},+\infty\right)$, there exist both global solutions and blow-up solutions. As for the extinction exponent, the extinction case happens to the interval $\left(q_{1},+\infty\right)$, while for $q \in\left(0, q_{1}\right)$, there exists a non-extinction bounded solution for any nonnegative initial datum. Moreover, when the critical case $q=q_{1}$ is concerned, the other parameter $\lambda$ will play an important role. In other words, when $\lambda$ belongs to different interval $\left(0, \lambda_{1}\right)$ or $\left(\lambda_{1},+\infty\right)$, where $\lambda_{1}$ is the first eigenvalue of $p$-Laplacian equation with zero boundary value condition, the solution has completely different properties.
\end{abstract}

\section{Introduction}

In this paper, we consider the following doubly degenerate parabolic equation with source

$$
\begin{array}{ll}
u_{t}-\operatorname{div}\left(\left|\nabla u^{m}\right|^{p-2} \nabla u^{m}\right)=\lambda u^{q}, & (x, t) \in \Omega \times(0,+\infty), \\
u(x, t)=0, & (x, t) \in \partial \Omega \times(0,+\infty), \\
u(x, 0)=u_{0}(x), & x \in \Omega,
\end{array}
$$

where $p>1, m>0, m(p-1)<1, \lambda>0, q>0, \Omega \subset \mathbb{R}^{N}(N>p)$ is a bounded domain with smooth boundary, and $u_{0}(x) \in C_{0}(\Omega)$ is a nonnegative function. Throughout this paper, I denote $Q=\Omega \times(0,+\infty), Q_{T}=\Omega \times(0, T), Q_{\left(t_{1}, t_{2}\right)}=$ $\Omega \times\left(t_{1}, t_{2}\right)$.

Received September 19, 2008.

2000 Mathematics Subject Classification. 35K50, 35K55, 35K65, 35B33.

Key words and phrases. non-Newtonian polytropic equation, critical blow-up exponent, critical extinction exponent. 
Parabolic equations like (1.1) appear in population dynamics, chemical reactions, heat transfer, and so on. In particular, equation (1.1) may be used to describe the non-stationary flows in a porous medium of fluids under polytropic conditions. In this case, equation (1.1) is called the non-Newtonian polytropic filtration equations (see [5], [8], [11] and references therein).

The problem of determining critical exponent is an interesting one in the general theory of blow-up or extinction solutions to different nonlinear evolution equations of mathematical physics (see [1], [3], [6], [7], [10] and references therein). Recently, a lot of papers are devoted to discuss the following degenerate equation

$$
\begin{array}{ll}
u_{t}=\left(\left|\left(u^{m}\right)_{x}\right|^{p-2}\left(u^{m}\right)_{x}\right)_{x}, & x>0, t>0, \\
-\left|\left(u^{m}\right)_{x}\right|^{p-2}\left(u^{m}\right)_{x}(0, t)=u^{q}(0, t), & t>0, \\
u(x, 0)=u_{0}(x), & x>0 .
\end{array}
$$

In [9], Wang et. al. considered (1.2) with $m>1, p>2, q \geq 0$. By constructing various self-similar upper solutions and lower solutions, they obtain the critical global existence exponent and critical Fujita exponent as $q_{0}=(m+1)(p-$ $1) / p, \quad q_{c}=(m+1)(p-1)$. In [4], Jin et. al. consider (1.2) with $m>0,1+1 /(m+$ $1) \leq p<1+1 / m, q>0$. They also get the critical global existence exponent and critical Fujita exponent as $q_{0}=(m+1)(p-1) / p, \quad q_{c}=(m+1)(p-1)$. Furthermore, they get if $0<q<m(p-1)$, then any nontrivial solution does not go extinction for any nontrivial and nonnegative initial value.

The motivation of this paper is what happens if the problem (1.2) with source and without boundary flux and to reveal the phenomena described as [4], [9]. Following from [2], [8], [11], we can get the local existence of weak solutions in the sense of the following definition.

Definition. A function $u \in E$ is called to be a weak upper (lower) solution of problem (1.1), provided for any $T>0$ and any $0 \leq \varphi \in \tilde{E}$, the following inequalities hold:

$$
\begin{gathered}
\iint_{Q_{T}} u_{t} \varphi d x d t+\iint_{Q_{T}}\left|\nabla u^{m}\right|^{p-2} \nabla u^{m} \nabla \varphi d x d t \geq(\leq) \lambda \iint_{Q_{T}} u^{q} \varphi d x d t, \\
\left.u\right|_{\partial \Omega \times(0, T)} \geq(\leq) 0, \quad u(x, 0) \geq(\leq) u_{0}(x), \quad \text { where } \\
E=\left\{u \in L^{2 q}\left(Q_{T}\right) \cap L^{2}\left(Q_{T}\right), u_{t} \in L^{2}\left(Q_{T}\right), \nabla u^{m} \in L^{p}\left(Q_{T}\right)\right\} \\
\tilde{E}=\left\{u \in L^{2}\left(Q_{T}\right),\left.u\right|_{\partial \Omega \times(0, T)}=0, u_{t} \in L^{2}\left(Q_{T}\right), \nabla u \in L^{p}\left(Q_{T}\right)\right\} .
\end{gathered}
$$

Furthermore, $u$ is called a weak solution of (1.1) if it is both a weak upper and a lower solution.

To state our results, we introduce the following two functions:

$$
F(u)=\frac{1}{p} \int_{\Omega}\left|\nabla u^{m}\right|^{p} d x-\frac{m \lambda}{q+m} \int_{\Omega} u^{q+m} d x, \quad H(u)=\frac{1}{m+1} \int_{\Omega} u^{m+1} d x,
$$


and the following eigenvalue problem for $p$-Laplace equation in $\Omega^{\prime} \supset \Omega$

$$
\begin{array}{ll}
-\operatorname{div}\left(|\nabla u|^{p-2} \nabla u\right)=\lambda u, & x \in \Omega^{\prime}, \\
u(x, t)=0, & x \in \partial \Omega^{\prime} .
\end{array}
$$

Denote $\lambda^{*}$ and $\varphi^{*}$ be the first eigenvalue and its corresponding eigenfunction of problem (1.3). It is obvious that $\lambda^{*}>0$ and $\varphi^{*}>0$ in $\Omega^{\prime}$, so $\varphi^{*}>0$ in $\bar{\Omega}$. Let

$$
M^{*}=\max _{x \in \bar{\Omega}} \varphi^{*}(x)>0, \quad m^{*}=\max _{x \in \bar{\Omega}} \varphi^{*}(x)>0 .
$$

Theorem 1.1. Let $u(x, t)$ be a weak solution of problem (1.1) :

(1) If $0<q<1$, then $u(x, t)$ exists globally, and is locally uniformly bounded;

(2) If $m(p-1)<q, F\left(u_{0}\right) \leq 0$ and $H\left(u_{0}\right)>0$, then $\lim _{t \rightarrow+\infty}\|u(\cdot, t)\|_{L^{\infty}(\Omega)}$ $=+\infty$;

(3) If $q<m(p-1)$, then $u(x, t)$ is globally uniformly bounded;

(4) If $q=m(p-1)$, then $u(x, t)$ is globally uniformly bounded if $\lambda$ is small enough such that $\lambda^{*} m^{*} \geq \lambda M^{* q / m}$;

(5) If $q>m(p-1)$, then $u(x, t)$ is globally uniformly bounded if the initial data $u_{0}$ is small enough such that $\delta \varphi^{*^{1} / m} \geq u_{0}$, where $\delta$ is some constant such that $\lambda^{*} m^{*} \geq \lambda \delta^{q-m(p-1)} M^{* q / m}$;

(6) If $q=1, F\left(u_{0}\right) \leq 0$ and $H\left(u_{0}\right)>0$, then $\lim _{t \rightarrow+\infty} H(u(t))=+\infty$;

(7) If $q>1, F\left(u_{0}\right) \leq 0$ and $H\left(u_{0}\right)>0$, then there exists $T^{*}$ with $0<T^{*}<$ $+\infty$ such that $\lim _{t \rightarrow T^{*}} H(u(t))=+\infty$, i.e., $u(x, t)$ blows up in finite time;

(8) If $q \geq 1$, then $u(x, t)$ is globally uniformly bounded if the initial data $u_{0}$ is small enough such that $\delta \varphi^{*^{1} / m} \geq u_{0}$, where $\delta$ is some constant such that $\lambda^{*} m^{*} \geq \lambda \delta^{q-m(p-1)} M^{* q / m}$.

Theorem 1.2. Let $\lambda_{1}$ be the first eigenvalue of the p-Laplacian equation with homogeneous Dirichlet boundary value condition. If $q \geq 1$ or $1>q>m(p-1)$, then any bounded and nonnegative weak solution of problem (1.1) vanishes in finite time for appropriate small initial datum $u_{0}(x)$. In addition, if $q=m(p-$ 1) with $\lambda<\lambda_{1}$, then any bounded and nonnegative weak solution of problem (1.1) vanishes in the sense of $\|\cdot\|_{L^{m+1}(\Omega)}$ as $t \rightarrow+\infty$, and in particular, if $p \geq(m N+N) /(N m+m+1)$ or $1<p<(m N+N) /(N m+m+1)$ with $\lambda<\gamma \lambda_{1} m^{p-1}(p /(\gamma+m(p-1)))^{p}$, where $\gamma>\max \{0,(N+N m-N m p-p) / p\}$, then $u$ vanishes in finite time too.

Remark 1.3. In fact, from the proof of Theorem 1.2, we see that when $q=1$, the boundness restriction on the solution of $u$ is unnecessary.

Theorem 1.4. Let $\lambda_{1}$ be the first eigenvalue of the p-Laplacian equation with homogeneous Dirichlet boundary value condition. For any nonnegative initial 
datum $u_{0}(x)$, problem (1.1) admits at least one bounded nonnegative and nonextinction weak solution for the case of $q=m(p-1)$ with $\lambda>\lambda_{1}$ or $0<q<$ $m(p-1)$.

Remark 1.5. From Theorems 1.1, 1.2, 1.4, we get the $q_{1}, q_{2}$ in the abstract is $q_{1}=m(p-1), q_{2}=1$.

Remark 1.6. Although we consider the case $m(p-1)<1$, we prefer to give some short note for the case of $m(p-1)>1$, which reveals a sharp difference from the case we considered: let $u$ be a nonnegative weak solution of the problem (1.1) with $m(p-1)>1$, then $u$ cannot vanish in finite time for any $q>0$ (see the note in Section 3). In particularly, without any change of the proof (3), (5) about Theorem 1.1, we can get that: If $q<m(p-1)$, then $u(x, t)$ is globally uniformly bounded; If $q>m(p-1), F\left(u_{0}\right) \leq 0$ and $H\left(u_{0}\right)>0$, then there exists $T^{*}$ with $0<T^{*}<+\infty$ such that $\lim _{t \rightarrow T^{*}} H(u(t))=+\infty$, i.e., $u(x, t)$ blows up in finite time.

This paper is organized as follows. In the next section, we give the critical blow-up exponent and prove Theorem 1.1. In Section 3, we give the critical extinction exponent and prove Theorems 1.2 and 1.4.

\section{Critical blow-up exponent}

In this section, we consider the critical blow-up exponent to problem (1.1) and prove Theorem 1.1.

Proof of Theorem 1.1. (1) Suppose $u$ is a weak solution of problem (1.1), then by the weak maximum principle (see [2], [8], [11], [13]), we conclude that

$$
\|u\|_{L^{\infty}\left(Q_{t}\right)} \leq\left\|u_{0}\right\|_{L^{\infty}(\Omega)}+t \lambda\|u\|_{L^{\infty}\left(Q_{t}\right)}^{q} .
$$

Since $0<q<1$, then it is not difficult to see that $u$ is locally uniformly bounded.

(2) Note the definition of $F(u)$ and $H(u)$, and a simple calculation show that

$$
\frac{d F(u)}{d t}=-m \int_{\Omega} u^{m-1}\left(u_{t}\right)^{2} d x \leq 0,
$$

$$
\frac{d H(u)}{d t}=-\int_{\Omega}\left|\nabla u^{m}\right|^{p} d x+\lambda \int_{\Omega} u^{q+m} d x=\left(1-\frac{m p}{q+m}\right) \int_{\Omega} \lambda u^{q+m} d x-p F(u) .
$$

According to $(2.2)$, we see that $F\left(u_{0}\right) \leq 0$ implies that $F(u) \leq 0$. Therefore we get

$$
\frac{d H(u)}{d t} \geq \lambda\left(1-\frac{m p}{q+m}\right) \int_{\Omega} u^{q+m} d x
$$


While for the case $m(p-1)<q<1$, if $u$ is a weak solution of problem (1.1), then by the weak maximum principle (see [2], [8], [11], [13]), we conclude that

$$
\|u\|_{L^{\infty}\left(Q_{t}\right)} \leq\left\|u_{0}\right\|_{L^{\infty}(\Omega)}+t \lambda\|u\|_{L^{\infty}\left(Q_{t}\right)}^{q},
$$

which implies that $u$ is bounded in any finite time. However, we have

$$
\lim _{t \rightarrow+\infty}\|u(\cdot, t)\|_{L^{\infty}(\Omega)}=+\infty .
$$

Suppose to the contrary, there would exist a positive constant $M$, such that $\|u(\cdot, t)\|_{L^{\infty}(\Omega)} \leq M$. Then

$$
\int_{\Omega} u^{1+m} d x \leq M^{1-q} \int_{\Omega} u^{q+m} d x .
$$

From (2.4), we infer that

$$
\frac{d H(u(t))}{d t} \geq \lambda(m+1)\left(1-\frac{m p}{q+m}\right) M^{q-1} H(u(t)) .
$$

Since $q+m>m p$, then $\lim _{t \rightarrow+\infty} H(u(t))=+\infty$.

(3) Let $\ell=\sup _{x \in \Omega} u_{0}(x)$ and define

$$
K_{0}=\max \left\{\ell,\left(\frac{\lambda m}{\lambda_{1}}\left(\frac{p m+1-m}{p m}\right)^{p}\right)^{1 /(m(p-1)-q)}\right\},
$$

where $\lambda_{1}$ is the first eigenvalue of the $p$-Laplacian equation with homogeneous Dirichlet boundary value condition and denote $A_{h}(t)=\{x \in \Omega ; u(x, t) \geq h\}$. In the following, we shall show that

$$
\|u(\cdot, t)\|_{L^{\infty}(\Omega)} \leq K_{0} .
$$

Multiplying equation $(1.1)$ by $\left(u-K_{0}\right)+$ yields

$$
\frac{1}{2} \frac{d}{d t} \int_{\Omega}\left(u-K_{0}\right)_{+}^{2} d x+\int_{\Omega}\left|\nabla u^{m}\right|^{p-2} \nabla u^{m} \nabla\left(u-K_{0}\right)_{+} d x=\lambda \int_{\Omega} u^{q}\left(u-K_{0}\right)_{+} d x .
$$

A simple computation, we get

$$
\begin{aligned}
& \int_{\Omega}\left|\nabla u^{m}\right|^{p-2} \nabla u^{m} \nabla\left(u-K_{0}\right)_{+} d x \\
= & \int_{A_{K_{0}}(t)}\left|\nabla u^{m}\right|^{p-2} \nabla u^{m} \nabla u d x \\
= & \frac{1}{m} \int_{A_{K_{0}}(t)} u^{1-m}\left|\nabla u^{m}\right|^{p} d x \\
= & \frac{1}{m}\left(\frac{p m}{p m+1-m}\right)^{p} \int_{A_{K_{0}}(t)}\left|\nabla u^{m+(1-m) / p}\right|^{p} d x .
\end{aligned}
$$

Take

$$
\tilde{u}= \begin{cases}u, & \text { if } u \geq K_{0}, \\ 0, & \text { otherwise }\end{cases}
$$


By using the Poincare's inequality, we get

$$
\int_{\Omega}\left|\nabla \tilde{u}^{m+(1-m) / p}\right|^{p} d x \geq \lambda_{1} \int_{\Omega} \tilde{u}^{m p+1-m} d x,
$$

i.e.,

$$
\int_{A_{K_{0}}(t)}\left|\nabla u^{m+(1-m) / p}\right|^{p} d x \geq \lambda_{1} \int_{A_{K_{0}}(t)} u^{m p+1-m} d x .
$$

Take (2.10) into account, we conclude that

$$
\begin{aligned}
& \frac{1}{2} \frac{d}{d t} \int_{\Omega}\left(u-K_{0}\right)_{+}^{2} d x+\frac{\lambda_{1}}{m}\left(\frac{p m}{p m+1-m}\right)^{p} \int_{A_{K_{0}}(t)} u^{m p+1-m} d x \\
\leq & \lambda \int_{\Omega} u^{q}\left(u-K_{0}\right)_{+} d x .
\end{aligned}
$$

Furthermore, we get

$$
\begin{aligned}
& \frac{1}{2} \frac{d}{d t} \int_{\Omega}\left(u-K_{0}\right)_{+}^{2} d x+\frac{\lambda_{1}}{m}\left(\frac{p m}{p m+1-m}\right)^{p} \int_{\Omega} u^{m(p-1)}\left(u-K_{0}\right)_{+} d x \\
\leq & \lambda \int_{\Omega} u^{q}\left(u-K_{0}\right)_{+} d x .
\end{aligned}
$$

Recalling the definition of $K_{0}$, we arrive at

$$
\begin{aligned}
& \frac{1}{2} \frac{d}{d t} \int_{\Omega}\left(u-K_{0}\right)_{+}^{2} d x \\
\leq & \int_{\Omega} u^{q}\left(u-K_{0}\right)_{+}\left(\lambda-\frac{\lambda_{1}}{m}\left(\frac{p m}{p m+1-m}\right)^{p} u^{m(p-1)-q}\right) d x \leq 0 .
\end{aligned}
$$

Noticing that $\int_{\Omega}\left(u-K_{0}\right)_{+}^{2} d x=0$, and combining with (2.17) we conclude that $\int_{\Omega}\left(u-K_{0}\right)_{+}^{2} d x=0$, which implies (2.9) holds.

(4) It is easy to see $\bar{u}=\delta \varphi^{*^{1 / m}}$ is an upper solution of (1.1), where $\delta$ is large enough such that $\delta \varphi^{*^{1 / m}} \geq u_{0}(x)$. The conclusion follows by comparison principle.

(5) Similar to (4), we can see $\bar{u}=\delta \varphi^{*^{1 / m}}$ is an upper solution of (1.1), where $\delta$ is some constant such that $\lambda^{*} m^{*} \geq \lambda \delta^{q-m(p-1)} M^{* q / m}$. The conclusion follows by comparison principle.

(6) When $q=1$, it follows from (2.4) that

$$
\frac{d H(u(t))}{d t} \geq \lambda(m+1)\left(1-\frac{m p}{1+m}\right) H(u(t)) .
$$

By a direct calculation, we obtain

$$
H(u(t)) \geq H\left(u_{0}\right) e^{((m+1) \lambda(1-m p /(1+m))) t} .
$$

Since $1+m>m p$, we have $\lim _{t \rightarrow+\infty} H(u(t))=+\infty$. 
(7) If $q>1$, recalling Höder's inequality, we get

$$
\int_{\Omega} u^{q+m} d x \geq C\left(\frac{1}{m+1} \int_{\Omega} u^{m+1} d x\right)^{(q+m) /(m+1)} .
$$

Thus, we get from (2.4) that

$$
\frac{d H(u(t))}{d t} \geq C \lambda\left(1-\frac{m p}{q+m}(H(u))\right)^{(q+m) /(m+1)} .
$$

Since $q+m>m p$ and $q>1$, there must exists a positive constant $T^{*}<+\infty$ such that $\lim _{t \rightarrow T^{*}} H(u(t))=+\infty$.

(8) Similar to (4), we can see $\bar{u}=\delta \varphi^{*^{1 / m}}$ is an upper solution of (1.1), where $\delta$ is some constant such that $\lambda^{*} m^{*} \geq \lambda \delta^{q-m(p-1)} M^{* q / m}$. The conclusion follows by comparison principle. The proof of Theorem 1.1 is complete.

\section{Critical extinction exponent}

Now, we characterize the critical extinction exponent of problem (1.1) and prove Theorems 1.2 and 1.4 .

Proof of Theorem 1.2. We divide the proof into three cases:

Case 1. $q \geq 1$. Let $M=\|u\|_{L^{\infty}(Q)}$. Multiplying equation (1.1) by $u^{\gamma}(\gamma>$ $m(1-p))$, and integrating over $\Omega$, we get

$$
\begin{aligned}
& \frac{1}{\gamma+1} \frac{d}{d t} \int_{\Omega} u^{\gamma+1} d x+\gamma m^{p-1}\left(\frac{p}{\gamma+m(p-1)}\right)^{p} \int_{\Omega}\left|\nabla u^{\frac{\gamma+m(p-1)}{p}}\right|^{p} d x \\
= & \lambda \int_{\Omega} u^{\gamma+q} d x \leq \lambda \int_{\Omega} M^{q-1} u^{\gamma+1} d x .
\end{aligned}
$$

It is clear that the inequality is valid for $q=1$ even if $u$ is unbounded. Since $N>p$, recalling the imbedding theorem, we have

$$
\begin{aligned}
& \int_{\Omega}\left|\nabla u^{\frac{\gamma+m(p-1)}{p}}\right|^{p} d x \\
\geq & C\left(\int_{\Omega} u^{\frac{N(\gamma+m(p-1))}{N-p}} d x\right)^{(N-p) / N} \\
\geq & C\left(\int_{\Omega} u^{\gamma+1} d x\right)^{(\gamma+m(p-1)) /(\gamma+1)},
\end{aligned}
$$

where $\gamma>\max \{0, m(1-p),(N+N m-N m p-p) / p\}$. To simplify, we denote

$$
f(t)=\int_{\Omega} u^{\gamma+1} d x, \quad \alpha=(\gamma+m(p-1)) /(\gamma+1)<1 .
$$

Hence, we have

$$
f^{\prime}(t)+C f^{\alpha}(t) \leq \lambda(\gamma+1) M^{q-1} f(t)
$$


If there exists a $t_{0}>0$, such that $f\left(t_{0}\right)=0$, then

$$
f(t) \leq \lambda(\gamma+1) M^{q-1} \int_{t_{0}}^{t} f(\tau) d \tau
$$

Recalling Gronwall's inequality, we obtain

$$
f(t) \equiv 0 \text { for any } t>t_{0} .
$$

Otherwise, $f(t)>0$ holds for all $t$. Then we get

$$
\left(f^{1-\alpha}\right)^{\prime}-\lambda(1-\alpha)(\gamma+1) M^{q-1} f^{1-\alpha} \leq-C(1-\alpha) .
$$

By a simple calculation, we arrive at

$$
f^{1-\alpha}(t)
$$

$$
\leq f^{1-\alpha}(0) e^{\lambda(1-\alpha)(\gamma+1) M^{q-1} t}-\frac{C}{\lambda(\gamma+1) M^{q-1}}\left(e^{\lambda(1-\alpha)(\gamma+1) M^{q-1} t}-1\right)
$$

$$
\leq\left(\left(\int_{\Omega} u_{0}^{\gamma+1} d x\right)^{1-\alpha}-\frac{C}{\lambda(\gamma+1) M^{q-1}}\right) e^{\lambda(1-\alpha)(\gamma+1) M^{q-1} t}+\frac{C}{\lambda(\gamma+1) M^{q-1}} .
$$

Let $u_{0}(x)$ be sufficiently small such that

$$
\left(\int_{\Omega} u_{0}^{\gamma+1} d x\right)^{1-\alpha} \leq \frac{C}{2 \lambda(\gamma+1) M^{q-1}}
$$

The two inequalities above give

$$
f^{1-\alpha}(t) \leq-\frac{C}{2 \lambda(\gamma+1) M^{q-1}}\left(e^{\lambda(1-\alpha)(\gamma+1) M^{q-1} t}-2\right) .
$$

From (3.10) above, we see that there must exist a $T>0$ such that $f^{1-\alpha}(t) \leq 0$ for $t \geq T$. Obviously, it is a contradiction. In conclusion, there exists a positive constant $T^{*}$ such that

$$
\int_{\Omega} u^{\gamma+1} d x \equiv 0 \text { for any } t \geq T^{*} .
$$

Case 2. $1>q>m(p-1)$. Multiplying equation (1.1) by $u^{\gamma}(\gamma>m(1-p))$, and integrating over $\Omega$, we get

$$
\begin{aligned}
& \frac{1}{\gamma+1} \frac{d}{d t} \int_{\Omega} u^{\gamma+1} d x+\gamma m^{p-1}\left(\frac{p}{\gamma+m(p-1)}\right)^{p} \int_{\Omega}\left|\nabla u^{\frac{\gamma+m(p-1)}{p}}\right|^{p} d x \\
= & \lambda \int_{\Omega} u^{\gamma+q} d x \leq \lambda|\Omega|^{(1-q) /(\gamma+1)}\left(\int_{\Omega} u^{1+\gamma} d x\right)^{(\gamma+q) /(\gamma+1)} .
\end{aligned}
$$

Choosing $\gamma>\max \{0, m(1-p),(N+N m-N m p-p) / p\}$. Then, according to the imbedding theorem $(N>p)$, we have

$$
\int_{\Omega}\left|\nabla u^{\frac{\gamma+m(p-1)}{p}}\right|^{p} d x \geq C_{1}\left(\int_{\Omega} u^{\gamma+1} d x\right)^{(\gamma+m(p-1)) /(\gamma+1)} .
$$


Substituting (3.13) into (3.12), we obtain

$$
\begin{aligned}
& \frac{1}{\gamma+1} \frac{d}{d t} \int_{\Omega} u^{\gamma+1} d x+\gamma C_{1} m^{p-1}\left(\frac{p}{\gamma+m(p-1)}\right)^{p}\left(\int_{\Omega} u^{\gamma+1} d x\right)^{(\gamma+m(p-1)) /(\gamma+1)} \\
\leq & \lambda|\Omega|^{(1-q) /(\gamma+1)}\left(\int_{\Omega} u^{1+\gamma} d x\right)^{(\gamma+q) /(\gamma+1)}
\end{aligned}
$$

By a direct calculation, we further have

$$
\begin{aligned}
& \frac{1}{1-m(p-1)} \frac{d}{d t}\left(\int_{\Omega} u^{1+\gamma} d x\right)^{(1-m(p-1)) /(\gamma+1)} \\
\leq & \lambda|\Omega|^{(1-q) /(\gamma+1)}\left(\int_{\Omega} u^{1+\gamma} d x\right)^{(q-m(p-1)) /(\gamma+1)}-\gamma C_{1} m^{p-1}\left(\frac{p}{\gamma+m(p-1)}\right)^{p} .
\end{aligned}
$$

To simplify, we denote

$$
\begin{aligned}
& \mu(u(t)) \\
= & \lambda|\Omega|^{(1-q) /(\gamma+1)}\left(\int_{\Omega} u^{1+\gamma} d x\right)^{(q-m(p-1)) /(\gamma+1)}-\gamma C_{1} m^{p-1}\left(\frac{p}{\gamma+m(p-1)}\right)^{p} .
\end{aligned}
$$

If $\mu\left(u_{0}\right)<0$, we claim that $\mu(u(t))$ is decreasing with $t$. To see this, we assume there exists some time $T>0$, such that $\left.\frac{d}{d t} \mu(u(t))\right|_{t=T} \geq 0$ and make a contradiction. Since $\mu\left(u_{0}\right)<0$, it is easy to see that $\left.\frac{d}{d t}\left(\int_{\Omega} u^{1+\gamma} d x\right)\right|_{t=0}<0$ from (3.15). So $\left.\frac{d}{d t} \mu(u(t))\right|_{t=0}<0$. Then there exist first positive time $T^{\prime} \leq T$ such that $\mu\left(u\left(T^{\prime}\right)\right)<\mu\left(u_{0}\right)<0$ and $\left.\frac{d}{d t} \mu(u(t))\right|_{t=T^{\prime}}=0$, i.e.,

$$
\left.\lambda|\Omega|^{\frac{1-q}{\gamma+1}} \frac{q-m(p-1)}{\gamma+1}\left(\int_{\Omega} u^{1+\gamma} d x\right)^{\frac{q-m(p-1)-\gamma-1}{\gamma+1}} \frac{d}{d t}\left(\int_{\Omega} u^{1+\gamma} d x\right)\right|_{t=T^{\prime}}=0
$$

If $u\left(x, T^{\prime}\right) \equiv 0$, we complete our proof. If $u\left(x, T^{\prime}\right) \not \equiv 0$, we get

$$
\left.\frac{d}{d t}\left(\int_{\Omega} u^{1+\gamma} d x\right)\right|_{t=T^{\prime}}=0
$$

Then, using (3.15), we get

$$
0=\left.\frac{1}{1-m(p-1)} \frac{d}{d t}\left(\int_{\Omega} u^{1+\gamma} d x\right)^{(1-m(p-1)) /(\gamma+1)}\right|_{t=T^{\prime}} \leq \mu\left(u\left(T^{\prime}\right)\right)<0 .
$$

We can get a contradiction, so the claim is correct. 

i.e.,

We further get $\frac{1}{1-m(p-1)} \frac{d}{d t}\left(\int_{\Omega} u^{1+\gamma} d x\right)^{(1-m(p-1)) /(\gamma+1)} \leq \mu\left(u_{0}\right)$ from (3.15),

$$
\begin{aligned}
& \left(\int_{\Omega} u^{1+\gamma} d x\right)^{(1-m(p-1)) /(\gamma+1)} \\
\leq & (1-m(p-1)) \mu\left(u_{0}\right) t+\left(\int_{\Omega} u_{0}^{1+\gamma} d x\right)^{(1-m(p-1)) /(\gamma+1)} .
\end{aligned}
$$

It is evident that there exists a positive constant $T^{*}>T^{\prime}$ such that

$$
\int_{\Omega} u^{1+\gamma} d x \equiv 0 \text { for all } t \geq T^{*} .
$$

Case 3. $q=m(p-1), \lambda<\lambda_{1}$. Multiplying equation (1.1) by $u^{m}$ and integrating over $\Omega$, we conclude

$$
\frac{1}{m+1} \frac{d}{d t} \int_{\Omega} u^{m+1} d x+\int_{\Omega}\left|\nabla u^{m}\right|^{p} d x \leq \lambda \int_{\Omega} u^{m p} d x .
$$

Note that $\lambda_{1} \int_{\Omega} u^{m p} d x \leq \int_{\Omega}\left|\nabla u^{m}\right|^{p} d x$, then we have

$$
\frac{1}{m+1} \frac{d}{d t} \int_{\Omega} u^{m+1} d x \leq-\left(\lambda_{1}-\lambda\right) \int_{\Omega} u^{m p} d x .
$$

Let $\ell_{0}=\|u\|_{L^{\infty}(Q)}, v=u / \ell_{0}$, so, $0 \leq v \leq 1$. Then we have $v^{m p} \geq v^{m+1}$ for $m p<m+1($ since $m(p-1)<1)$ and

$$
\begin{aligned}
\frac{d}{d t} \int_{\Omega} v^{m+1} d x & \leq-(m+1) \ell_{0}^{m p-m-1}\left(\lambda_{1}-\lambda\right) \int_{\Omega} v^{m p} d x \\
& \leq-(m+1) \ell_{0}^{m p-m-1}\left(\lambda_{1}-\lambda\right) \int_{\Omega} v^{m+1} d x,
\end{aligned}
$$

which implies that

$$
\int_{\Omega} v^{m+1} d x \leq e^{-(m+1) \ell_{0}^{m p-m-1}\left(\lambda_{1}-\lambda\right) t} \int_{\Omega} v_{0}^{m+1} d x .
$$

Therefore, we conclude that $\|u(\cdot, t)\|_{L^{m+1}(\Omega)} \rightarrow 0$ as $t \rightarrow+\infty$. In addition, by (3.19), we have

$$
\frac{1}{m+1} \frac{d}{d t} \int_{\Omega} u^{m+1} d x+\int_{\Omega}\left|\nabla u^{m}\right|^{p} d x \leq \frac{\lambda}{\lambda_{1}} \int_{\Omega}\left|\nabla u^{m}\right|^{p} d x .
$$

Using the imbedding theorem, we obtain if $p \geq(m N+N) /(N m+m+1)$

$$
\begin{aligned}
\frac{1}{m+1} \frac{d}{d t} \int_{\Omega} u^{m+1} d x & \leq-\left(1-\frac{\lambda}{\lambda_{1}}\right) \int_{\Omega}\left|\nabla u^{m}\right|^{p} d x \\
& \leq-C_{0}\left(1-\frac{\lambda}{\lambda_{1}}\right)\left(\int_{\Omega} u^{m+1} d x\right)^{m p /(m+1)}
\end{aligned}
$$


i.e.,

$$
\begin{aligned}
\left(\int_{\Omega} u^{m+1} d x\right)^{(m+1-m p) /(m+1)} \leq & \left(\int_{\Omega} u_{0}^{m+1} d x\right)^{(m+1-m p) /(m+1)} \\
& -C_{0}(m+1-m p)\left(1-\frac{\lambda}{\lambda_{1}}\right) t .
\end{aligned}
$$

So there exists a positive constant $T^{*}$, such that

$$
\int_{\Omega} u^{m+1} d x \equiv 0 \text { for any } t \geq T^{*},
$$

which implies $u$ vanishes in finite time.

The following argument is devoted to the discussion of the case of $1<p<$ $(m N+N) /(N m+m+1)$. Similar to (3.1), we obtain

$$
\begin{aligned}
& \frac{1}{\gamma+1} \frac{d}{d t} \int_{\Omega} u^{\gamma+1} d x+\gamma m^{p-1}\left(\frac{p}{\gamma+m(p-1)}\right)^{p} \int_{\Omega}\left|\nabla u^{\frac{\gamma+m(p-1)}{p}}\right|^{p} d x \\
= & \lambda \int_{\Omega} u^{\gamma+m(p-1)} d x \leq \frac{\lambda}{\lambda_{1}} \int_{\Omega}\left|\nabla u^{\frac{\gamma+m(p-1)}{p}}\right|^{p} d x,
\end{aligned}
$$

i.e.,

$$
\begin{aligned}
& \frac{1}{\gamma+1} \frac{d}{d t} \int_{\Omega} u^{\gamma+1} d x \\
\leq & -\left(\gamma m^{p-1}\left(\frac{p}{\gamma+m(p-1)}\right)^{p}-\frac{\lambda}{\lambda_{1}}\right) \int_{\Omega}\left|\nabla u^{\frac{\gamma+m(p-1)}{p}}\right|^{p} d x .
\end{aligned}
$$

Since $\lambda<\gamma \lambda_{1} m^{p-1}\left(\frac{p}{\gamma+m(p-1)}\right)^{p}$ and $\gamma>\max \{0,(N+N m-N m p-p) / p\}$, then according to the imbedding theorem, we further have

$$
\begin{aligned}
& \frac{1}{\gamma+1} \frac{d}{d t} \int_{\Omega} u^{\gamma+1} d x \\
\leq & -C\left(\gamma m^{p-1}\left(\frac{p}{\gamma+m(p-1)}\right)^{p}-\frac{\lambda}{\lambda_{1}}\right)\left(\int_{\Omega} u^{\gamma+1} d x\right)^{(\gamma+m(p-1)) /(\gamma+1)} .
\end{aligned}
$$

Since $(\gamma+m(p-1)) /(\gamma+1)<1$, then a similar argument as before gives that there exists a $T^{*}>0$ such that

$$
\int_{\Omega} u^{\gamma+1} d x \equiv 0 \text { for any } t \geq T^{*} .
$$

The proof of Theorem 1.2 is complete.

Proof of Theorem 1.4. The main method of the proof of Theorem 1.4 is constructing a pair of ordered upper and lower solution $(\bar{u}(x, t), \underline{u}(x, t))(\bar{u}(x, t) \geq$ $\underline{u}(x, t))$ with $\underline{u}(x, t)$ is non-extinction, then the problem exists a weak solution $u(x, t)$ satisfying $\underline{u}(x, t) \leq u(x, t) \leq \bar{u}(x, t)$ (see [2], [8], [11]) and we get the 
results. Firstly, we construct weak lower non-extinction solution of problem (1.1).

Case 1. $q=m(p-1), \lambda>\lambda_{1}$, let $\varphi_{1}(x)$ is the corresponding eigenfunction with $\left\|\varphi_{1}(x)\right\|_{L^{\infty}(\Omega)}=1$. Take $\underline{u}(x, t)=g(t) \varphi_{1}(x)$, where $g(t)$ satisfies

$$
g^{\prime}(t)=\left(\lambda-\lambda_{1}\right) g^{m(p-1)}, g(t)>0 \text { for } t>0, \quad g(0)=0 .
$$

Hence, we get

$$
\begin{aligned}
\underline{u}_{t}=\left(\lambda-\lambda_{1}\right) \varphi_{1} g^{m(p-1)} & \leq\left(\lambda-\lambda_{1}\right) \varphi_{1}^{m(p-1)} g^{m(p-1)} \\
& \leq \operatorname{div}\left(\left|\nabla \underline{u}^{m}\right|^{p-2} \nabla \underline{u}^{m}\right)+\lambda v^{m(p-1)} .
\end{aligned}
$$

That is $\underline{u}$ is a weak lower solution of problem (1.1).

Case 2. $q<m(p-1)$, let $\underline{u}(x, t)=\mu g(t) \varphi_{1}(x)$, where $g(t)$ is a solution of the following problem

$$
g^{\prime}(t)=-\lambda_{1} g^{m(p-1)}+\lambda g^{q}, g(t)>0 \text { for } t>0, \quad g(0)=0 .
$$

Then we have

$$
\begin{gathered}
\underline{u}_{t}=\left(-\lambda_{1} g^{m(p-1)}+\lambda g^{q}\right) \mu \varphi_{1}, \\
\operatorname{div}\left(\left|\nabla \underline{u}^{m}\right|^{p-2} \nabla \underline{u}^{m}\right)+\lambda \underline{u}^{q}=-\lambda_{1} \mu^{m(p-1)} g^{m(p-1)} \varphi_{1}^{m(p-1)}+\lambda \mu^{q} g^{q} \varphi_{1}^{q} .
\end{gathered}
$$

Then we can choose a $\mu>0$ small enough such that

$$
\lambda_{1} g^{m(p-1)}\left(\mu^{m(p-1)} \varphi_{1}^{m(p-1)}-\mu \varphi_{1}\right) \leq \lambda g^{q}\left(\mu^{q} \varphi_{1}^{q}-\mu \varphi_{1}\right) .
$$

In fact, from (3.33), it is not difficult to see that $g(t)$ is a nondecreasing and bounded function. Let $M=\max _{\lambda>0} \lambda / \lambda_{1} g^{m(p-1)-q}$. In addition, let $F(x)=$ $\left(x^{q}-x\right) /\left(x^{m(p-1)}-x\right)$, it is easy to check that $F(x)$ is decreasing in $(0,1)$ and $\lim _{x \rightarrow 0^{+}} F(x)=+\infty, \lim _{x \rightarrow 1^{-}} F(x)=q(1-q) /(m(p-1)(1-m(p-1)))$. Thus we can choose $\mu>0$ small enough to satisfy (3.36). Next we construct an upper weak solution of problem (1.1). Let $\bar{u}(x, t)$ be the solution of the following problem

$$
\begin{array}{ll}
\bar{u}_{t}-\operatorname{div}\left(\left|\nabla \bar{u}^{m}\right|^{p-2} \nabla \bar{u}^{m}\right)=\lambda\left(\bar{u}_{+}+1\right)^{q}, & (x, t) \in \Omega \times(0,+\infty), \\
\bar{u}(x, t)=0, & (x, t) \in \partial \Omega \times(0,+\infty), \\
\bar{u}(x, 0)=u_{0}(x) \geq 0, & x \in \Omega .
\end{array}
$$

From [2], [8], [11], we know that problem (3.37) admits at least one weak solution. In addition, the weak solution $\bar{u}(x, t)$ is also a weak upper solution of problem (1.1). The following is devoted to prove $\underline{u}(x, t) \leq \bar{u}(x, t)$. From the 
definition of $\underline{u}(x, t)$ and $\bar{u}(x, t)$, we deduce

$$
\begin{aligned}
& \iint_{Q_{t}}(\underline{u}-\bar{u})_{t} \varphi d x d \tau+\iint_{Q_{t}}\left(\left|\nabla \underline{u}^{m}\right|^{p-2} \nabla \underline{u}^{m}-\left|\nabla \bar{u}^{m}\right|^{p-2} \nabla \bar{u}^{m}\right) \nabla \varphi d x d \tau \\
\leq & \lambda \iint_{Q_{t}}\left(\underline{u}^{q}-\left(\bar{u}_{+}+1\right)^{q}\right) \varphi d x d \tau \text { for all } 0 \leq \varphi \in \tilde{E} .
\end{aligned}
$$

Take $\varphi=(\underline{u}-\bar{u})_{+}$, then we arrive at

$$
\begin{aligned}
& \frac{1}{2} \int_{\Omega}(\underline{u}-\bar{u})_{+}^{2} d x+\iint_{Q_{t}}\left(\left|\nabla \underline{u}^{m}\right|^{p-2} \nabla \underline{u}^{m}-\left|\nabla \bar{u}^{m}\right|^{p-2} \nabla \bar{u}^{m}\right) \nabla(\underline{u}-\bar{u})_{+} d x d \tau \\
\leq & \lambda \iint_{Q_{t}}\left(\underline{u}^{q}-\left(\bar{u}_{+}+1\right)^{q}\right)(\underline{u}-\bar{u})_{+} d x d \tau \\
\leq & \lambda q \iint_{Q_{t}}\left(\underline{u}-\left(\bar{u}_{+}+1\right)\right)(\underline{u}-\bar{u})_{+} d x d \tau \\
\leq & \lambda q \iint_{Q_{t}}(\underline{u}-\bar{u})_{+}^{2} d x d \tau .
\end{aligned}
$$

Recalling Gronwall's inequality, we get

$$
\int_{\Omega}(\underline{u}-\bar{u})_{+}^{2} d x=0 \quad \text { for all } t>0
$$

which implies that $\underline{u} \leq \bar{u}$ a.e. in $Q$. Hence problem (1.1) admits a weak solution $\underline{u} \leq u \leq \bar{u}$. Since $\underline{u}$ does not vanish, so does $u$. The proof of Theorem 1.4 is complete.

Note. We give the proof of Remark 1.6 in this note. The basic idea follows from [12]. If $u$ is a weak solution of (1.1), then $u$ is also a weak upper solution of the following problem

$$
\begin{array}{ll}
v_{t}=\operatorname{div}\left(\left|\nabla v^{m}\right|^{p-2} \nabla v^{m}\right), & (x, t) \in \Omega \times(0,+\infty), \\
v(x, t)=0, & (x, t) \in \partial \Omega \times(0,+\infty), \\
v(x, 0)=u_{0}(x), & x \in \Omega .
\end{array}
$$

The we can get the following property about $v(x, t)$.

Lemma 3.1. Let $v$ be a weak solution of problem (3.41). Then we have

(1) If $m(p-1)>1$, then

$$
\frac{\partial v}{\partial t} \geq-\frac{v}{(m(p-1)-1) t}
$$

in the sense of distributions.

(2) If $0<m(p-1)<1$, then

$$
\frac{\partial v}{\partial t} \leq \frac{v}{(1-m(p-1)) t}
$$


in the sense of distributions.

Proof. Denote

$$
v_{r}(t)=r v\left(x, r^{m(p-1)-1} t\right)
$$

for all $(x, t) \in Q$ and all $r \in(1 / 2,1)$. Clearly, $v_{r}$ is a weak solution of equation (3.41) with the following initial-boundary condition

$$
\left.v_{r}(x, t)\right|_{\partial \Omega \times(0,+\infty)}=0, \quad v_{r}(x, 0)=r u_{0}(x), \forall x \in \Omega .
$$

Noting $r \in(1 / 2,1)$ and using (3.41) and (3.45) we get

(3.46) $\left.v_{r}(x, t)\right|_{\partial \Omega \times(0,+\infty)}=\left.v(x, t)\right|_{\partial \Omega \times(0,+\infty)}=0, \quad v_{r}(x, 0) \leq u_{0}(x), \forall x \in \Omega$.

Applying the comparison principle (see [2], [8], [11]) we have

$$
v_{r}(x, t) \leq v(x, t), \quad \forall(x, t) \in Q .
$$

(i) For $m(p-1)>1$, by $(3.47)$, we get

$$
\frac{(v(x, \varrho t))^{m(p-1)-1}-(v(x, t))^{m(p-1)-1}}{\varrho t-t} \geq \frac{(1 / \varrho-1)(v(x, t))^{m(p-1)-1}}{\varrho t-t},
$$

where $\varrho=r^{m(p-1)-1}$. Letting $\varrho \rightarrow 1^{-}$, we get

$$
\frac{\partial}{\partial t}(v(x, t))^{m(p-1)-1} \geq-\frac{1}{t}(v(x, t))^{m(p-1)-1}
$$

in distribution, which implies that (1) holds.

(ii) For $0<m(p-1)<1$, by $(3.47)$, we have

$$
\frac{(v(x, \varrho t))^{1-m(p-1)}-(v(x, t))^{1-m(p-1)}}{\varrho t-t} \leq \frac{(\varrho-1)(v(x, t))^{1-m(p-1)}}{\varrho t-t},
$$

where $\varrho=r^{m(p-1)-1}$. Letting $\varrho \rightarrow 1^{+}$, we get

$$
\frac{\partial}{\partial t}(v(x, t))^{1-m(p-1)} \leq \frac{1}{t}(v(x, t))^{1-m(p-1)}
$$

in distribution, which implies that (2) holds. The proof of Lemma 3.1 is complete.

Now we can demonstrate the weak solution of (1.1) can not vanish in finite time if $m(p-1)>1$. The (1) of Lemma 3.1 tells us that $\frac{\partial\left(t^{1 /(m(p-1)-1)} v\right)}{\partial t} \geq 0$, which means that

$$
\operatorname{supp} v(\cdot, s) \subset \operatorname{supp} v(\cdot, t) \text { for all } s, t \text { with } 0<s<t .
$$

Since $u$ is a weak upper solution of problem (3.41), then by the comparison principle (see [2], [8], [11], [13]), we have $u(x, t) \geq v(x, t)$, which implies that $u(x, t)$ does not vanish in finite time.

Acknowledgments. We would thank the referees for their valuable comments and suggestions. The first author is supported by Natural Science Foundation Project of China SWU, SWU208029, the second author is supported by NNSF 
of China (10771226) and in part by Natural Science Foundation Project of CQ CSTC (2007BB0124).

\section{References}

[1] K. Deng and H. A. Levine, The role of critical exponents in blow-up theorems: the sequel, J. Math. Anal. Appl. 243 (2000), no. 1, 85-126.

[2] E. DiBenedetto, Degenerate Parabolic Equations, Springer-Verlag, New York, 1993.

[3] H. Fujita, On the blowing up of solutions to the cauchy problems for $u_{t}=\Delta u+u^{1+\alpha}$, Journal of the Faculty of Science University of Tokyo Section 1Mathematics Astronomy Physics Chemistry 13 (1996), 109-124.

[4] C. H. Jin and J. X. Yin, Critical exponents and non-extinction for a fast diffusive polytropic filtration equation with nonlinear boundary sources, Nonlinear Anal. 67 (2007), no. $7,2217-2223$.

[5] A. S. Kalashnikov, Some problems of the qualitative theory of second-order nonlinear degenerate parabolic equations, Uspekhi Mat. Nauk 42 (1987), no. 2, 135-176.

[6] Y. C. Kwong, Boundary behavior of the fast diffusion equation, Trans. Amer. Math. Soc. 322 (1990), no. 1, 263-283.

[7] H. A. Levine, The role of critical exponents in blowup theorems, SIAM Rev. 32 (1990), no. 2, 262-288.

[8] J. L. Vázquez, The Porous Medium Equations: Mathematical Theory, Oxford Univ. Press, 2007.

[9] Z. J. Wang, J. X. Yin, and C. P. Wang, Critical exponents of the non-Newtonian polytropic filtration equation with nonlinear boundary condition, Appl. Math. Lett. 20 (2007), no. 2, 142-147.

[10] M. Winkler, A strongly degenerate diffusion equation with strong absorption, Math. Nachr. 277 (2004), 83-101.

[11] Z. Q. Wu, J. N. Zhao, J. X. Yin, and H. L. Li, Nonlinear Diffusion Equations, World Scientific Publishing Co., Inc., River Edge, NJ, 2001.

[12] H. J. Yuan, Extinction and positivity for the evolution p-Laplacian equation, J. Math. Anal. Appl. 196 (1995), no. 2, 754-763.

[13] H. J. Yuan, S. Z. Lian, W. J. Gao, X. J. Xu, and C. L. Cao, Extinction and positivity for the evolution p-Laplace equation in $\mathbb{R}^{N}$, Nonlinear Anal. 60 (2005), no. 6, 1085-1091.

JuN ZHOU

SCHOOL OF MATHEMATICS AND STATISTICS

Southwest University

Chongqing, 400715, P. R. China

E-mail address: zhoujun_math@hotmail.com

Chunlai Mu

College of Mathematics AND Physics

Chongqing University

Chongling, 400044, P. R. China

E-mail address: Chunlaimu@yahoo.com.cn 